\title{
Aromatase Inhibition Therapy
}

National Cancer Institute

\section{Source}

National Cancer Institute. Aromatase Inhibition Therapy. NCI Thesaurus. Code C15525.

Drug treatment to block the action of aromatases, thereby reducing estrogen biosynthesis. 